\title{
Association between sitting time and non-alcoholic fatty liver disease in South Korean population: a cross-sectional study: a cross-sectional study
}

Jae Hong Joo

Yonsei University

Hwi Jun Kim

Yonsei University

Eun-Cheol Park

Yonsei University College of Medicine

Sung-In Jang ( $\square$ jangsi@yuhs.ac)

Yonsei University College of Medicine https://orcid.org/0000-0002-0760-2878

Research article

Keywords: NAFLD, hepatic steatosis index, sitting time, physical activity, obesity

Posted Date: March 26th, 2020

DOI: https://doi.org/10.21203/rs.3.rs-18598/v1

License: (c) (i) This work is licensed under a Creative Commons Attribution 4.0 International License.

Read Full License 


\section{Abstract}

Background: To examine the association between sitting time and non-alcoholic fatty liver disease among South Koreans aged $\geq 20$ years.

Methods : Data from the 2016-2018 Korea National Health and Nutrition Examination Survey were used for the analysis. Non-alcoholic fatty liver disease was diagnosed according to a hepatic steatosis index of $>36$. Sitting time was categorized into as $<5,5-7,8-10$, and $>10$ hours. Multiple logistic regression analysis was used to examine the association between sitting time and non-alcoholic fatty liver disease in South Korean population.

Results : A total of 13,518 participants were enrolled. The odds for having NAFLD in $5-7,8-10$, and > 10 sitting hours were 1.04 (Cl: 0.85-1.28), 1.23(Cl: 1.01-1.51), and 1.33(Cl: 1.10-1.62), respectively. The odds ratio increased in magnitude with longer hours of sitting time (test for trend: P-value $=0.0009$ ).

Conclusion : Advising physical exercises and discouraging sedentary activities may help to alleviate NAFLD among the South Korean population.

\section{Background}

Non-alcoholic fatty liver disease (NAFLD) is among the most common liver disorders, with approximately $9-30 \%$ of the population in developed countries having NAFLD ${ }^{1,2}$. Fatty liver is often associated with heavy alcohol intake, but it may also occur in the absence of alcohol. The impact of globalization has led to a more westernized lifestyle and an increased frequency of obesity, and this contributed to an increased prevalence of NAFLD in the Asian countries ${ }^{3,4}$. The incidence of NAFLD is projected to increase due to the prevalence of sedentary behavior. If left untreated, NAFLD leads to end-stage liver disease. Thus, NAFLD is a serious public health burden that needs to be addressed and managed immediately ${ }^{5}$.

Based on an evolutionary perspective, moving and engaging in every manner of manual labor was essential to survival of our species ${ }^{6,7}$. However, technological advances have created environments where sedentary lifestyle is encouraged and this is an increasing public health concern ${ }^{8}$. The prevalence of sedentary behavior is increasing in modern societies, and adults are becoming less active throughout the day ${ }^{9}$. Sedentary behavior involves sitting or lying down and not sufficiently spending energy substantially during the awake time ${ }^{10}$. There is increasing evidence that excessive sitting time is associated with higher risk for adverse health outcomes such as cardiovascular disease, type 2 diabetes, cancer, and mortality ${ }^{11-13}$. Maintaining balanced energy expenditure and physical activity is a key aspect of healthy behavior; thus, sedentary lifestyle should not be considered lightly.

A positive relationship between sedentary behavior and NAFLD has been reported persistently. NAFLD is closely linked to obesity, insulin resistance, and metabolic disorders, all of which are associated with excessive sedentary behavior ${ }^{14,15}$. The purpose of this study was to examine the association between 
sitting time and NAFLD in the South Korean population and to elucidate whether the prolonged sitting time plays a potential role in developing NAFLD.

\section{Methods}

\section{Study participants}

Data were obtained from the 2016-2018 Korea National Health and Nutrition Examination Survey (KNHANES), which was conducted by the Korea Centers for Disease Control and Prevention. The KNHANES is a self-report survey conducted in Koreans of all age and is designed to gather annual national data on sociodemographic, economic, and health-related conditions and behaviors. The survey is consisted of three components (health interview, health examination and nutrition survey), all of which are conducted by trained staff members including physicians and medical technicians ${ }^{16}$.

Of the 24,269 survey participants, we excluded who tested positive for serologic markers for liver disease (hepatitis $B$, hepatitis $C$, or liver cirrhosis) $(n=735)$, were aged $<20$ years who did not undergo blood testing conducted by the KNHANES ( $n=6,868)$, and were not representative of covariates considered in the study (failed to answer the survey questionnaires) $(n=3,148)$. Accordingly, the final sample size consisted of 13,518 participants (Fig. 1). This study was an analysis of existing data; thus it did not require approval by ethics review board. The data that was used in this study is the KNHANES and it has been getting an annual review and approval by Korea Centers for Disease Control (KCDC) Research Ethics Review Committee since 2007.

\section{NAFLD classification}

NAFLD is the main dependent variable in this study. NAFLD in this study was diagnosed according to the hepatic steatosis index (HSI), which was developed by the Department of Internal Medicine and Liver Research Institute in Seoul National University College of Medicine to efficiently select individuals for liver ultrasonography ${ }^{17}$. The HSI formula was derived via logistic regression model using serum alanine aminotransferase (ALT) to serum aspartate aminotransferase (AST) ratio, body mass index (BMI), and diabetes mellitus status: $\mathrm{HSI}=8 \times($ ALT/AST ratio $)+\mathrm{BMI}\left(+2\right.$, if female; + 2, if with diabetes mellitus) ${ }^{17}$. Participants were considered to have NAFLD if their HSI value was above 36.

\section{Sitting time}

The main independent variable is the participants' sitting time. Sitting time was measured by asking participants to report the following question adopted from the International Physical Activity Questionnaire (IPAQ) ${ }^{18}$. "How many hours did you spend time sitting per day during the last week?" This questionnaire indicates the time spent in academic and/or leisurely activities in a reclined or seated position. Participants' responses to sitting time were divided into 4 categories using quartiles: $<5,5-7$, $8-10$, and $>10$ hours.

\section{Covariates}


Sociodemographic, economic, and health-related factors were also considered in the study.

Sociodemographic factors included age, educational attainment, and marital status. Economic factors included household income and occupation. Health-related factors include sleeping time (hours), total energy intake ((carbohydrate $(\mathrm{g}) \times 4 \mathrm{kcal} / \mathrm{g})+($ protein $(\mathrm{g}) \times 4 \mathrm{kcal} / \mathrm{g})+(\mathrm{fat}(\mathrm{g}) \times 9 \mathrm{kcal} / \mathrm{g}))$, physical activeness (active: $\geq 150 \mathrm{~min}$ of moderate activity, $\geq 75 \mathrm{~min}$ of vigorous activity, or a mixture of both for $\geq 150 \mathrm{~min}$; inactive: $<150 \mathrm{~min}$ of moderate activity, $<75 \mathrm{~min}$ of vigorous activity, or a mixture of both for $<150 \mathrm{~min}$ ), pack years of smoking, current drinking status, comorbidity of hypertension, and comorbidity of diabetes mellitus.

\section{Statistical analysis}

The frequencies and percentages of participants were calculated for each of the categorized variables included in the study. The variables included in the analysis were all categorical, those that were not initially categorical were converted into categories (age, BMI, total energy intake, etc.). The chi-square $\left(\chi^{2}\right)$ test was performed to assess the chi-square differences between the groups within each categorized variable (Table 1). Multiple logistic regression analysis was used to calculate the odds ratios (with 95\% confidence intervals) for NAFLD according to the participants' report on sitting time (Table 2). The subgroup analysis for NAFLD stratified by the participants' sex, physical activeness, and obesity status defined by BMI was also performed using multiple logistic regression (Fig. 2).

The reported odds ratios were adjusted for all covariates considered in the study. The sampling weight variables were applied in the analysis to improve the representativeness of the sample. KNHANES has constructed sample weights to take into account survey non-response, over-sampling, post-stratification, and sampling error. The use of sample weights in the analysis is recommended to produce an unbiased national estimate. For all data analysis, we used SAS version 9.4 (SAS Institute, Inc, Cary, NC, USA) and the significance level was set at $p$ value $<0.05$.

\section{Results}

The general characteristics of the study population are shown in Table 1. Of 13,518 participants, 3,140 (23.2\%) reported had NAFLD. The frequencies of having NAFLD varied across the different sitting hour categories. The participants who reported $>10$ hours of sitting per day had the highest frequency of having NAFLD (25.4\%), whereas those who reported $<5$ hours of sitting per day had the lowest frequency of having NAFLD (20.5\%) among the study sample.

Based on the odds ratios calculated from the multiple logistic analysis, the relationship between NAFLD and sitting time was analyzed along with other covariates. The participants who reported $<5$ sitting hours per day served as the reference group in this study and the relative risk for other 'sitting time' groups are expressed in Table 2. The odds for NAFLD in ' $5-7$ ', ' $8-10$ ', and '> 10 hours' were 1.04 (Cl: $0.85-1.28$ ), 1.23(Cl: 1.01-1.51), and 1.33(Cl: 1.10-1.62), respectively. The odds ratio increase in magnitude with longer hours of sitting time was tested for statistical significance using the linear trend test (test for trend: P-value $=0.0009$ ). 
Odds ratio for NAFLD according to the participants' sitting time was stratified by sex, physical activeness, and obesity status (Fig. 2). In this analysis, '< 5 hours' group were again served as the reference group. In both sexes, those who reported $>10$ hours of sitting per day had the relatively higher odds compared to those who reported shorter amount of sitting time (male 1.32, Cl: 0.99-1.76; female 1.33, Cl: 1.03-1.72). When participants were stratified by their physical activeness, the significant results were only observed in physically inactive group. Those who reported $>10$ hours of sitting per day had the highest odds (1.84, Cl: 1.42-2.38) within the physically inactive group and it was also the higher compared to their physically active courterparts. Moreover, the risk of having NAFLD was relatively high in those who reported 8-10 hours (1.28, Cl: 1.02-1.59) and > 10 hours of sitting time (1.33, Cl: 1.09-1.64) among the obese participants.

\section{Discussion}

\section{Main findings}

Our overall findings were that there is an association of sitting time with NAFLD and the risk of having NAFLD increases in magnitude with longer hours of sitting.

\section{What is already known?}

Sedentary behavior, which is characterized by a lack of muscle movement, directly affects cardiometabolic function ${ }^{6}$. The human body is designed to move, and previous studies reported that inactivity results in impaired lipid steatosis and insulin resistance ${ }^{19,20}$. This aligns with findings of our study which suggest that a higher rate of NAFLD was observed in sedentary participants. Prolonged sitting is correlated with higher BMI, body fat, and circulatory lipids, all of which are associated with liver lipid content ${ }^{21}$. Cumulative fat in the mitochondria, one of the key regulators of liver pathophysiology, progresses to non-alcoholic steatohepatitis with inflammation ${ }^{22}$. Mitochondrial dysfunction disrupts the encoding of cytochromes during carcinogenesis, and this leads to increased reactive oxygen species (ROS) production. Fat disposition in the liver may be because of ROS proliferation in Kupffer cells and hepatocytes ${ }^{23}$.

Previously, other studies resembling ours demonstrated a close relationship between sedentary lifestyle and NAFLD ${ }^{24-26}$. These studies suggested that prolonged sitting time and decreased physical activity level were positively associated with the prevalence of NAFLD, supporting the importance of reducing time spent sitting in addition to promoting physical activity. To date, lifestyle modification remains as one of the most important option in NAFLD management. The situation with respect to sedentary behavior, however, is remained widely unexplored.

\section{What this study adds}

\section{Analysis of the results}


In our study, the probability of having NAFLD was relatively high participants who were sedentary and physically inactive. Physical activity is the primary strategy for managing NAFLD. Gains in physical activity improves serum liver enzymes, reduces hepatic fatty infiltration, and reduces a degree of hepatic inflammation and fibrosis, all of which contribute to health benefits beyond the prevention of NAFLD 27 . The benefits of exercise and fitness have been supported by results of previous clinical trials. Normalized level of ALT and significant weight loss were observed in participants who increased their physical activity ${ }^{28,29}$. Regular physical activity is important because the frequency and intensity of physical activity are strong factors for predicting a person's hepatic outcome.

Primarily, NAFLD is a consequence of insulin resistance, and thus occurs frequently among obese participants ${ }^{30}$. Our results showed that the risk of developing NAFLD is higher in obese participants who sat for more than 8 hours per day than those who sat less than 5 hours a day. Obesity is associated with increased visceral fat that releases free fatty acids into the hepatic portal circulation and the concentration of free fatty acids serves as the key mediator of insulin resistance ${ }^{31}$. The absence of physical activity aggravates insulin sensitivity and glucose homeostasis. Sedentary behavior causes downregulation of insulin receptor in muscle tissue ${ }^{32,33}$. This means that prolonged sitting and physical inactivity impairs the delivery of insulin and glucose to the muscle and hence deteriorate the metabolism of free fatty acid ${ }^{34}$.

\section{Limitations and strengths of this study}

This study has several limitations. First, although HSI has been proven to have high sensitivity for detecting NAFLD (sensitivity of $93.1 \%$ and specificity of $92.4 \%$ ) ${ }^{17}$, there are still tiny odds of falsepositive or false negative. Second, this is study was a cross-sectional study and was unable to provide a causal relation between sitting time and NAFLD. Third, the KNHANES uses self-report questionnaires, and thus the data extracted may have been subject to recall bias. The strength of the study is that the dataset generated from the KNHANES is nationally representative of the health status in South Korea. KNHANES questionnaires are updated annually to incorporate the changes in the real-life health circumstances of South Koreans. KNHANES has been extremely useful in health-related studies and provides meaningful insights for South Korean health policies.

\section{Conclusion}

We found that sitting time was associated with NAFLD among the South Korean population aged > 20 years. The association was stronger for physically inactive participants. Further, there was also a positive association between NAFLD and obesity in conjunction with sedentary behavior. among female participants. Lifestyle modifications to engage in physical activity and reduce weight could contribute to managing and preventing NAFLD in the South Korean population. Advising exercise and discouraging sedentary activities may help to alleviate NAFLD in South Korea. Nonetheless, further studies are needed to validate the findings of our study and to examine a causal relationship between sitting time and the incidence of NAFLD. 


\section{Declarations}

Ethical approval and consent to participate: This study was an analysis of existing data; thus it did not require approval by ethics review board. The data that was used in this study is the KNHANES and it has been getting an annual review and approval by Korea Centers for Disease Control (KCDC) Research Ethics Review Committee since 2007.

Consent for publication: Not applicable.

Availability of data and material: The KNHANES was openly available in https://knhanes.cdc.go.kr/knhanes/index.do by submitting written oath and data utilization plan.

Competing interest: The authors declare that they have no competing interest.

Funding: This study was supported by a faculty research grand of Yonsei Univesity College of Medicine (6-2018-0174 and 6-2017-0157).

Author Contributions: JH.J., EC.P., and SI.J. designed the study, collected the data, performed the statistical analysis, and wrote the manuscript. H.K. contributed to the discussion and reviewed and edited the manuscript. SI.J. is the guarantor of this work and as such, had full access to all of the data. SI.J. assumes responsibility for the integrity of the data and the accuracy of the data analysis.

Acknowledgements: We thank the Korean Centers for Disease Control (KCDC) which produced and provided national level survey data. We also thank those colleagues of the Institute of Health Services Research in Yonsei University that have given advice for important intellectual content.

\section{Authors' information:}

Jae Hong Joo ${ }^{1,2}$; Eun-Cheol Park ${ }^{2,3}$; Hwijun Kim ${ }^{1,2}$; Doo Woong Lee ${ }^{1,2}$; Dong-Woo Choi ${ }^{1,2}$; Sung-In Jang ${ }^{2,3}$

${ }^{1}$ Department of Public Health, Graduate School, Yonsei University, Seoul, Republic of Korea

${ }^{2}$ Institute of Health Services Research, Yonsei University, Seoul, Republic of Korea

${ }^{3}$ Department of Preventive Medicine, Yonsei University College of Medicine, Seoul, Republic of Korea

\section{*Corresponding author: Sung-In Jang, MD, PhD}

Department of Preventive Medicine \& Institute of Health Services Research, Yonsei University College of Medicine

50 Yonsei-ro, Seodaemun-gu, Seoul 03722, Republic of Korea

Tel: +82-2-2228-1863; E-mail: JANGSI@yuhs.ac; Fax: +82-2-392-8133 


\section{References}

1. Armstrong MJ, Houlihan DD, Bentham L, et al. Presence and severity of non-alcoholic fatty liver disease in a large prospective primary care cohort. Journal of hepatology. 2012;56(1):234-240.

2. Cichoż-Lach H, Celiński K, Prozorow-Król B, Swatek J, Słomka M, Lach T. The BARD score and the NAFLD fibrosis score in the assessment of advanced liver fibrosis in nonalcoholic fatty liver disease. Medical Science Monitor: International Medical Journal of Experimental and Clinical Research. 2012;18(12):CR735-CR740.

3. Loomba R, Sanyal AJ. The global NAFLD epidemic. Nature Reviews: Gastroenterology \& Hepatology. 2013;10(11):686-690.

4. Lim YS, Kim WR. The global impact of hepatic fibrosis and end-stage liver disease. Clinics in liver disease. 2008;12(4):733-746, vii.

5. Lee Y-h, Kim SU, Song K, et al. Sarcopenia is associated with significant liver fibrosis independently of obesity and insulin resistance in nonalcoholic fatty liver disease: Nationwide surveys (KNHANES 2008-2011). Hepatology (Baltimore, Md). 2016;63(3):776-786.

6. Owen N, Sparling PB, Healy GN, Dunstan DW, Matthews CE. Sedentary behavior: emerging evidence for a new health risk. Mayo Clinic Proceedings. 2010;85(12):1138-1141.

7. Hill JO, Wyatt HR, Reed GW, Peters JC. Obesity and the environment: where do we go from here? Science. 2003;299(5608):853.

8. Dzewaltowski DA. Emerging technology, physical activity, and sedentary behavior. Exercise and Sport Sciences Review. 2008;36(4):171-172.

9. Knaeps S, Bourgois JG, Charlier R, Mertens E, Lefevre J, Wijndaele K. Ten-year change in sedentary behaviour, moderate-to-vigorous physical activity, cardiorespiratory fitness and cardiometabolic risk: independent associations and mediation analysis. British Journal of Sports Medicine. 2018;52(16):1063.

10. Tremblay M. Letter to the Editor: Standardized use of the terms "sedentary" and "sedentary behaviours". Applied Physiology, Nutrition, and Metabolism. 2012;37(3):540-542.

11. Proper KI, Singh AS, van Mechelen W, Chinapaw MJM. Sedentary behaviors and health outcomes among adults: A systematic review of prospective studies. American Journal of Preventive Medicine. 2011;40(2):174-182.

12. Thorp AA, Owen N, Neuhaus M, Dunstan DW. Sedentary behaviors and subsequent health outcomes in adults: A systematic review of longitudinal studies, 1996-2011. American Journal of Preventive Medicine. 2011;41(2):207-215.

13. van der Ploeg HP, Chey T, Korda RJ, Banks E, Bauman A. Sitting time and all-cause mortality risk in 222497 australian adults. Archives of Internal Medicine. 2012;172(6):494-500.

14. Zelber-Sagi S, Lotan R, Shlomai A, et al. Predictors for incidence and remission of NAFLD in the general population during a seven-year prospective follow-up. Journal of hepatology. 2012;56(5):1145-1151. 
15. Kumashiro N, Erion DM, Zhang D, et al. Cellular mechanism of insulin resistance in nonalcoholic fatty liver disease. Proceedings of the National Academy of Sciences of the United States of America. 2011;108(39):16381-16385.

16. Kweon S, Kim Y, Jang M-j, et al. Data resource profile: the Korea National Health and Nutrition Examination Survey (KNHANES). Int J Epidemiol. 2014;43(1):69-77.

17. Lee JH, Kim D, Kim HJ, et al. Hepatic steatosis index: a simple screening tool reflecting nonalcoholic fatty liver disease. Digestive and Liver Disease. 2010;42(7):503-508.

18. Oh JY, Yang YJ, Kim BS, Kang JHJJotKAoFM. Validity and Reliability of Korean Version of International Physical Activity Questionnaire (IPAQ) Short Form. 2007;28(7):532-541.

19. Ekblom Ö, Ekblom-Bak E, Rosengren A, Hallsten M, Bergström G, Börjesson M. Cardiorespiratory fitness, sedentary behaviour and physical activity are independently associated with the metabolic syndrome, results from the SCAPIS pilot study. PLOS ONE. 2015;10(6):e0131586.

20. Yates T, Khunti K, Wilmot EG, et al. Self-reported sitting time and markers of inflammation, insulin resistance, and adiposity. American Journal of Preventive Medicine. 2012;42(1):1-7.

21. Trenell MI. Sedentary behaviour, physical activity, and NAFLD: Curse of the chair. Journal of hepatology. 2015;63(5):1064-1065.

22. Gerber $L$, Otgonsuren $M$, Mishra $A$, et al. Non-alcoholic fatty liver disease (NAFLD) is associated with low level of physical activity: a population-based study. Alimentary of Pharmacology and Therapeutics. 2012;36(8):772-781.

23. Sato N. Central role of mitochondria in metabolic regulation of liver pathophysiology. Journal of Gasteroenterology and Hepatology. 2007;22(s1):S1-S6.

24. Fan J-G, Kim S-U, Wong VW-S. New trends on obesity and NAFLD in Asia. Journal of hepatology. 2017;67(4):862-873.

25. Ryu S, Chang Y, Jung H-S, et al. Relationship of sitting time and physical activity with non-alcoholic fatty liver disease. Journal of hepatology. 2015;63(5):1229-1237.

26. Hallsworth K, Thoma C, Moore S, et al. Non-alcoholic fatty liver disease is associated with higher levels of objectively measured sedentary behaviour and lower levels of physical activity than matched healthy controls. 2015;6(1):44-51.

27. Zelber-Sagi S, Ratziu V, Oren R. Nutrition and physical activity in NAFLD: an overview of the epidemiological evidence. World Journal of Gastroenterology. 2011;17(29):3377.

28. St George A, Bauman A, Johnston A, Farrell G, Chey T, George J. Independent effects of physical activity in patients with nonalcoholic fatty liver disease. Hepatology (Baltimore, Md). 2009;50(1):6876.

29. Sreenivasa Baba C, Alexander G, Kalyani B, et al. Effect of exercise and dietary modification on serum aminotransferase levels in patients with nonalcoholic steatohepatitis. Journal of Gasteroenterology and Hepatology. 2006;21(1 Pt 1):191-198.

30. Angulo P. Obesity and nonalcoholic fatty liver disease. Nutrition Reviews. 2007;65:S57-S63. 
31. Angulo P. NAFLD, obesity, and bariatric surgery. Gastroenterology. 2006;130(6):1848-1852.

32. Boule NG, Haddad E, Kenny GP, Wells GA, Sigal RJ. Effects of exercise on glycemic control and body mass in type 2 diabetes mellitus: a meta-analysis of controlled clinical trials. Jama. 2001;286(10):1218-1227.

33. Goodyear LJ, Kahn BB. Exercise, glucose transport, and insulin sensitivity. Annual review of medicine. 1998;49:235-261.

34. Hannukainen JC, Nuutila P, Borra R, et al. Increased physical activity decreases hepatic free fatty acid uptake: a study in human monozygotic twins. The Journal of physiology. 2007;578(Pt 1):347-358.

\section{Tables}

Due to technical limitations, the tables are only available as a download in the supplemental files section.

\section{Figures}




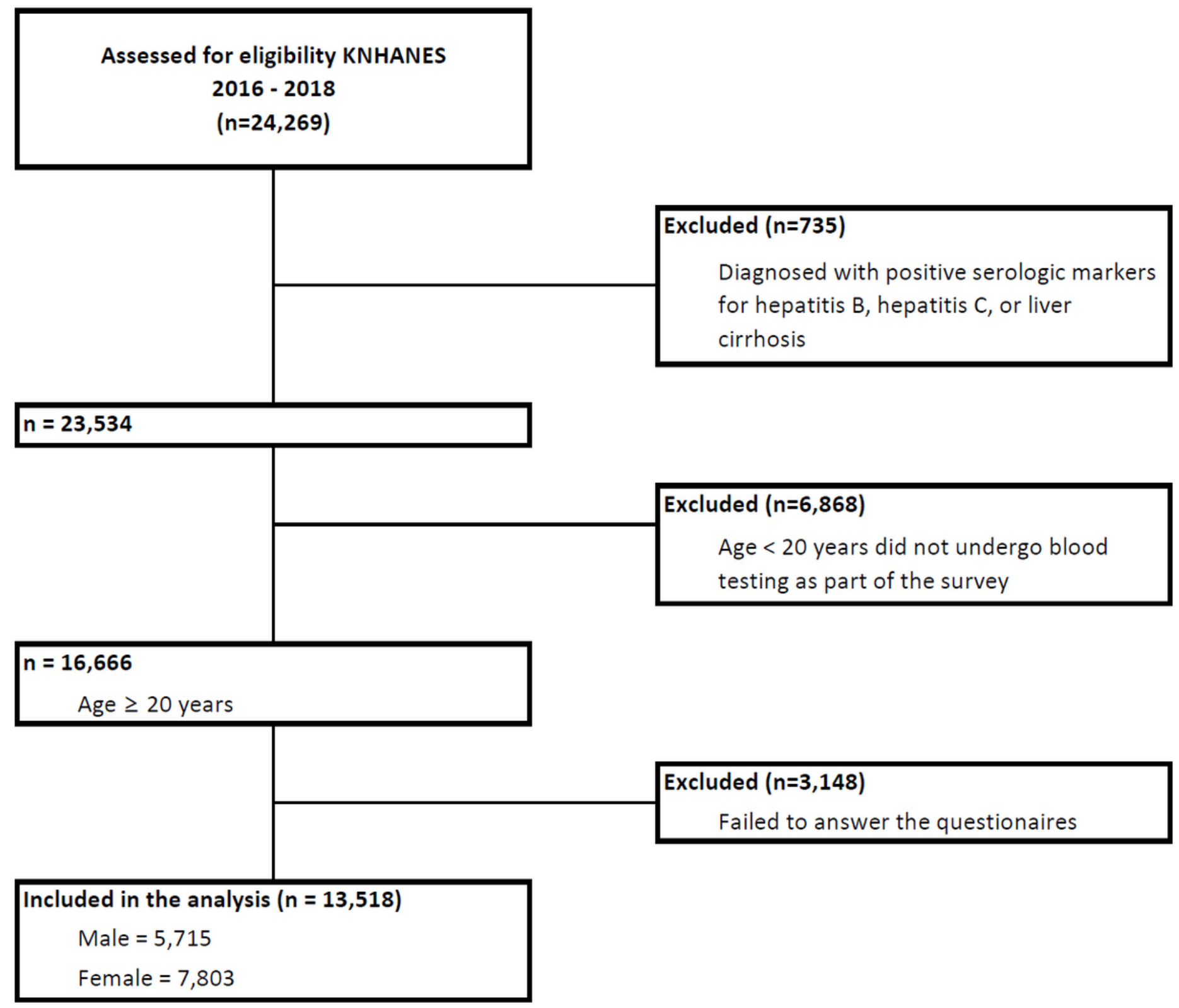

\section{Figure 1}

Flow diagram of subject inclusion and exclusion 


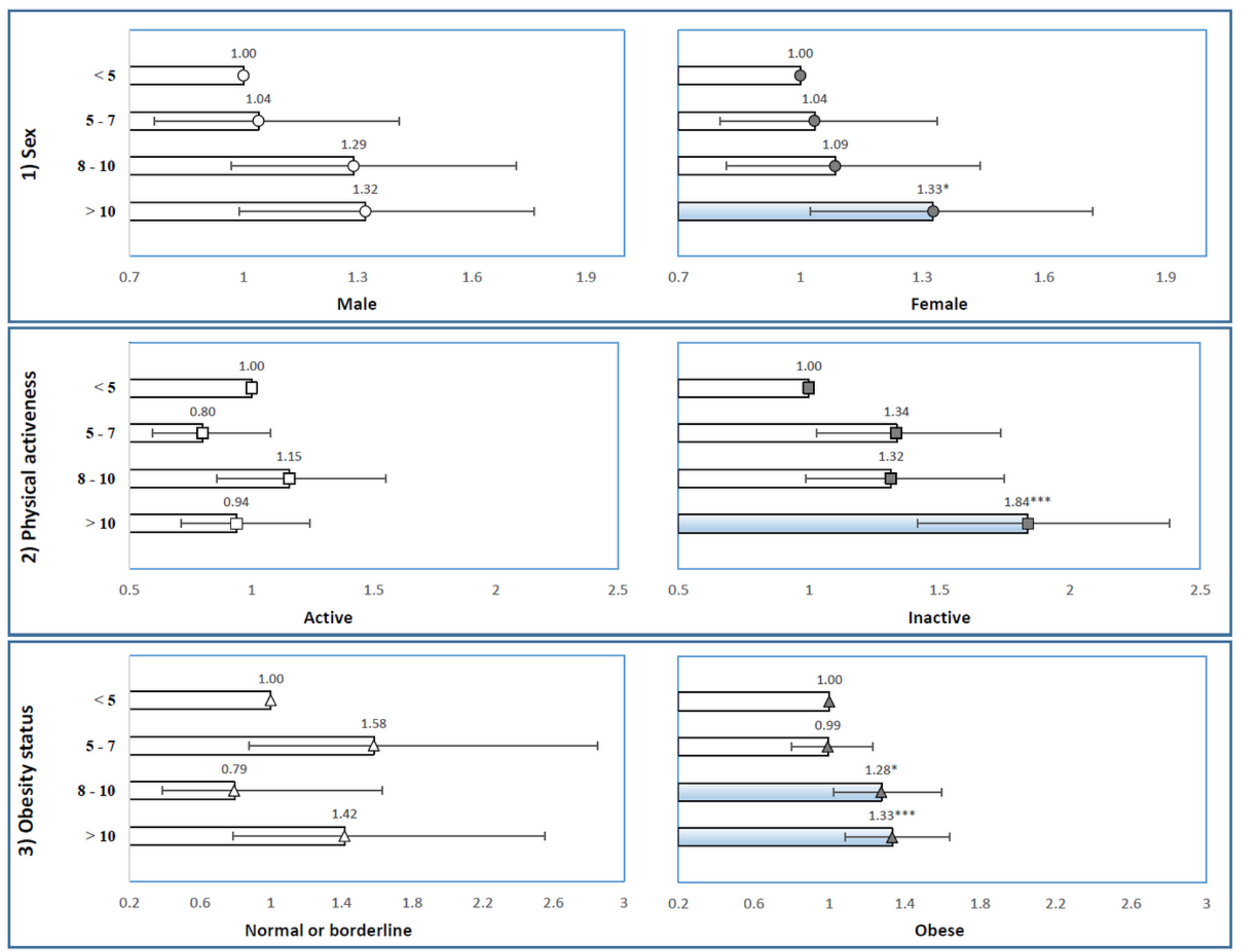

Figure 2

Forest plot representing odds ratio for NAFLD according to the participants' sitting time stratified by 1) sex, 2) physical a ct iveness, and 3) obesity status defined by $B M I * P$ value $<0.05, P$ value $<0.01$

\section{Supplementary Files}

This is a list of supplementary files associated with this preprint. Click to download.

- AssociationbetweensittingtimeandNAFLDinSouthKorea.xlsx 